\title{
Undulator system tolerance analysis for the European x-ray free-electron laser
}

\author{
Yuhui Li, Bart Faatz, and Joachim Pflueger \\ Deutsches Elektronen Synchrotron (DESY), Notkestraße 85, 22603 Hamburg, Germany
}

(Received 23 July 2008; published 7 October 2008)

\begin{abstract}
A detailed study of hardware related tolerances for the undulator system for the European x-ray freeelectron laser (XFEL) has been performed. Various realistic error scenarios were taken into account. These included girder deformation under magnetic loads, the influence of temperature variation, errors caused by limited accuracy of the motion control system, and phase matching errors. Undulator errors are classified into periodic and random errors. For periodic errors, such as girder deformation, a close and universal correlation between rms phase shake and power degradation was established, while the results of random errors show more scatter in the results, which have to be evaluated statistically. For XFEL parameters the correlation is very good and can be used to evaluate the influence of different error sources without the need to do extended FEL simulations. The method was applied to reevaluate some critical tolerances on the XFEL undulator systems: girder deformation, accuracy for gap control, and the requirement on temperature stability. The results show that these tolerances could be relaxed as compared to earlier work.
\end{abstract}

DOI: 10.1103/PhysRevSTAB.11.100701

PACS numbers: $41.60 . \mathrm{Cr}$

\section{INTRODUCTION}

The European x-ray free-electron laser (XFEL) will generate photons in the $\mathrm{x}$-ray range from 0.8 to $12.4 \mathrm{keV}$ $(1.6-0.1 \mathrm{~nm})[1]$ using the principle of self-amplified spontaneous emission (SASE) [2,3]. Simulations show that, in order to reach saturation in the above energy range, the length of the undulator needs to be longer than $200 \mathrm{~m}$ [1]. For practical reasons the undulator is subdivided into $5-\mathrm{m}$ long segments. In between segments there are so-called intersection units. Each is $1.1 \mathrm{~m}$ long and comprises a quadrupole for beam focusing, a phase shifter for adjusting the phase between the electrons and the light created in different undulator segments, a beam position monitor, synchrotron radiation absorbers, and vacuum pumps. An undulator segment and intersection form an undulator cell. It is therefore more appropriate to talk about an undulator system $[1,4]$. For example, the SASE1 undulator system for the European XFEL is comprised of 33 cells, each consisting of a $5 \mathrm{~m}$ long undulator segment and a $1.1 \mathrm{~m}$ long intersection. The system has a total length of $201.3 \mathrm{~m}$ [1], see also Table I.

There are various error sources that might affect FEL operation. Examples are: beam steering errors, quadrupole alignment errors, undulator alignment errors, external ambient fields, etcetera. These errors are external errors with respect to individual undulator segments. They are not considered in this paper. There are, however, errors that depend on details of the design of the undulator segments and the hardware used in the intersections. These errors result in a variation of the $K$ parameter along undulator segments. Conventional error analysis compares $K$-parameter variation to the FEL bandwidth, which is on the order of the Pierce parameter $\rho$. Since in the case of the
XFEL $\rho$ is about $10^{-4}$, very stringent requirements may result: The tolerable gap variation is limited to less than $1 \mu \mathrm{m}$ and the acceptable temperature variation inside the undulator system to less than $0.08^{\circ} \mathrm{C}$. These requirements are hard to fulfill and would need a substantial design effort and expensive solutions. Therefore, one objective of this paper is to do a more careful analysis in order to get a more realistic view on critical tolerances relevant for the hardware design of undulator systems.

We distinguish between two kinds of undulator field errors. The first is random, for example, because of the inhomogeneously magnetized magnet blocks. There are several examples found in the literature [5-11]. The second kind are errors that change periodically along the undulator. Here is one example: In order to limit total deformation under changing magnetic loads, equidistant support points are chosen, so that the total girder deflection is approximately periodic. Temperature variation along the undulator may be treated as a periodic problem as shown below. In this paper both error types are taken into account with special emphasis on the periodic ones.

There are steering and nonsteering errors [12-15]. Steering errors induce small local kicks in the trajectory that reduce the overlap between electron beam and the radiation field. Nonsteering errors perturb the ponderomotive phase and induce a phase shake between the electron and the photon field. Both errors can significantly increase the saturation length and reduce the FEL power.

Steering errors are easily detected in magnetic field measurements. Because of the stiff electron beam in the XFEL, however, these tolerances can be compensated using shimming and tuning procedures in a straightforward manner. In this paper, therefore, they are not considered, 
TABLE I. Parameters used for simulation.

\begin{tabular}{lcc}
\hline \hline Parameter & Value & Unit \\
\hline Undulator period $\lambda_{U}$ & 35.6 & $\mathrm{~mm}$ \\
Undulator gap & 10 & $\mathrm{~mm}$ \\
Peak field at 10 mm gap & 1.0 & $\mathrm{~T}$ \\
$K_{\text {peak }}$ at 10 mm gap & 3.3 & \\
$K=K_{\text {rms }}$ at 10 mm gap & 2.33 & \\
Segment length & 5 & $\mathrm{~m}$ \\
Intersection length & 1.1 & $\mathrm{~m}$ \\
Undulator cell length & 6.1 & $\mathrm{~m}$ \\
Number of cells & 33 & \\
Magnetic length & 165 & $\mathrm{~m}$ \\
System length & 201.3 & $\mathrm{~m}$ \\
Electron beam energy & 17.5 & $\mathrm{GeV}$ \\
Radiation wavelength & 0.1 & $\mathrm{~nm}$ \\
Energy spread & 1.5 & $\mathrm{MeV}$ \\
Bunch peak current & 5 & $\mathrm{kA}$ \\
Transverse normalized emittance & 1.4 & $\mathrm{~mm} \mathrm{mrad}$ \\
Average $\beta$ function & 32 & $\mathrm{~m}$ \\
\hline \hline
\end{tabular}

and only nonsteering errors are taken into account: thus the phase shake is the only cause for power degradation.

At the European XFEL there will be two undulator systems working in the hard $\mathrm{x}$-ray regime from 0.1 to $0.4 \mathrm{~nm}$. They are called SASE1 and SASE2. For a 17.5$\mathrm{GeV}$ beam SASE1 will work at a fixed-radiation wavelength of $0.1 \mathrm{~nm}$, while SASE2 is adjustable from 0.1 to $0.4 \mathrm{~nm}$. Simulations and results are very similar for SASE1 and SASE2. Therefore, for simplicity, this paper concentrates on SASE1. A complete study including SASE2 can be found in Ref. [16]. Table I lists the parameters for SASE1 and the XFEL used in the simulation. To prevent confusion throughout this paper the $K$ parameter follows the GENESIS 1.3 convention. For a sinusoidal field this means: $K=K_{\text {rms }}=K_{\text {peak }} / \sqrt{2}$.

\section{TOLERANCE ANALYSIS}

\section{A. Influence of phase shifter errors}

Inside an undulator segment there is a phase slippage of $2 \pi$ per undulator period between electrons and photons. In the field-free region inside the intersections, there is no field and the slippage is perturbed. This is of special importance if the gap is changed. To ensure that electrons and radiation preserve their ponderomotive phase at the end of each intersection, an adjustable magnetic chicane, called a phase shifter, is needed in each intersection. The phase change induced by the phase shifter is

$$
\phi=-2 \pi \frac{L_{D}\left(1+K_{D}^{2}\right)}{\lambda_{s}\left(2 \gamma^{2}\right)},
$$

where $L_{D}$ is the length of the phase shifter, $K_{D}$ is the phase shifter parameter, which is similar to undulator parameter,

$$
K_{D}=\frac{e}{m c} \sqrt{\left\langle A_{x}^{2}\right\rangle}=\frac{e}{m c} \sqrt{\frac{1}{L_{D}} \int_{0}^{L_{D}} A_{x}^{2}(z) d z}
$$

with $A_{x}$ the vector potential in the $x$ direction, $\nabla \times \vec{A}=\vec{B}$.

Ideally, $\phi$ is adjusted such that the phase advance for a whole undulator cell consisting of undulator segment plus intersection is an integer times $2 \pi$. This preserves the ponderomotive phase and guarantees maximum amplification of the optical field at the entrance of the next undulator cell. If the phase shifter is set incorrectly, a phase error $\Delta \phi$ is induced. The impact of a phase shifter error $\Delta \phi$ by changing the value of $K_{D}$ was investigated. For simplicity the absolute value of the error was kept constant over the system. If $\Delta \phi$ has the same sign for all cells of the undulator system, this is called unidirectional error. In contrast, for alternating errors the sign of $\Delta \phi$ alternates from one cell to the next.

Unidirectional phase shift errors continuously change the phase in one direction. This has the consequence that the radiation wavelength shifts. In this case the rms phase shake can be calculated by $\sigma_{\Delta \phi}=(1 / 2 \sqrt{3})|\Delta \phi|$ [16]. On the other hand, the optimized wavelength does not shift for alternating errors and its rms phase shake is given by $\sigma_{\Delta \phi}=|\Delta \phi / 2|$. This is $\sqrt{3}$ times larger than for unidirectional errors. Because the phase shake is related to power reduction, unidirectional errors are expected to give smaller power reduction than alternating error for a given value of $\Delta \phi$. The corresponding simulation results will be shown in the sections below.

\section{B. Phase shake of undulator related errors}

In the ideal case, the undulator gap is constant, and therefore the undulator $K$ parameter equals the design value and shows no variation. In reality, however, gap errors are unavoidable. For example, due to magnetic forces, the undulator girder deforms. For one specific gap, this error can be compensated using the pole-height adjustment method [17]. But for a different gap, the magnetic force, and thus girder deformation changes, resulting in field errors due to changing deformation. The XFEL undulators are designed with a fourfold support structure with support points separated by $1.2 \mathrm{~m}$ as required by Bessel's condition. This means that the deformation of the gap resulting from a uniformly acting magnetic force is decreased/increased in a symmetric fashion. The resulting deformation profile is approximately sinusoidal and periodic along the undulator system. This is shown in Fig. 1(a). In a similar fashion, there might be a gap taper as shown in Fig. 1(b) as well. Periodic sawtooth profiles will later be used to estimate the influence of temperature variations.

Other, different periodic deformation profiles such as sinus, sawtooth, triangular, piecewise constant, and parabolic profiles were investigated. While for some of these 
(a)
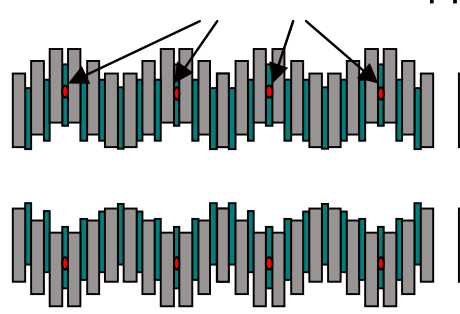

(b)



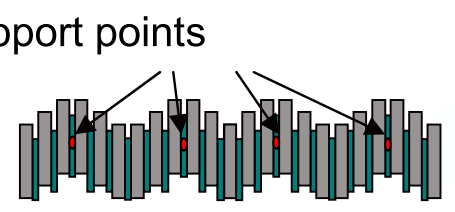
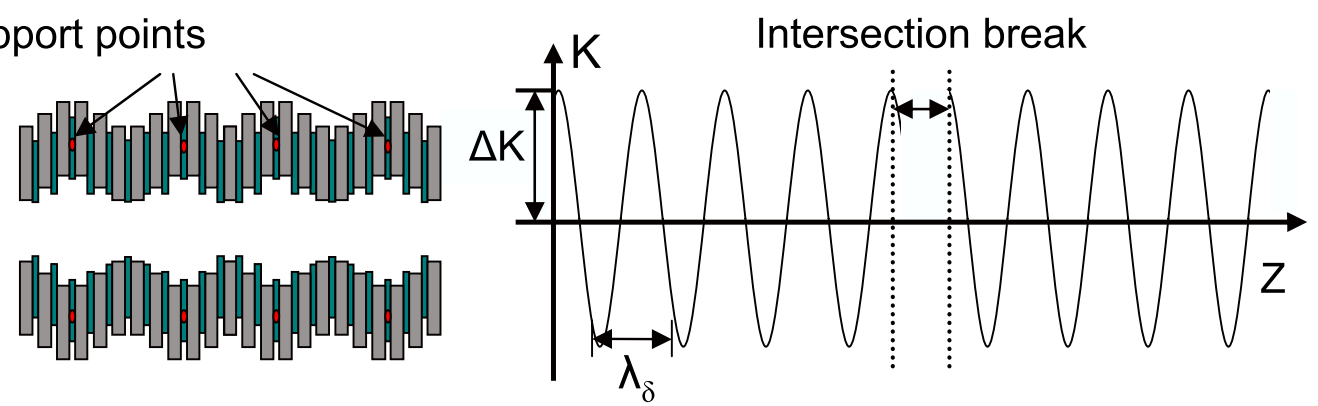
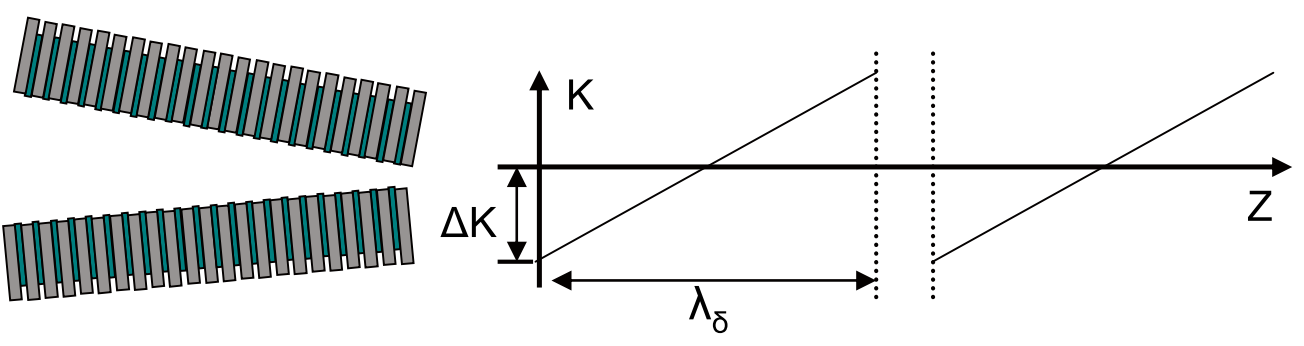

FIG. 1. (Color) Gap variation errors and the impact on the $K$ parameter. Part (a) shows the girder deformation due to magnetic forces. The girders of the XFEL undulators use a fourfold support at the Bessel points. The resulting deformation curve is symmetric and has an approximately sinusoidal shape. Part (b) shows the gap taper error.

profiles there are real applications, the others were primarily of academic interest.

For small changes the gap error and the resulting $K$ parameter variation are proportional. The right side of Fig. 1 shows the resulting variation of the $K$ parameter for sinusoidal and linear sawtooth gap variation. These profiles were used in the simulations.

First we analyze the impact of periodic errors on phase shake. A more complete analysis with theoretical derivation can be found in Ref. [16]. The field error is assumed to be much smaller than the field itself, $\Delta K \ll K_{0}$, where $\Delta K$ and $K_{0}$ denote the maximum error amplitude and the nominal value of the undulator parameter, respectively. The phase error $\Delta \varphi$ is

$$
\Delta \varphi(z)=\frac{k_{u}}{1+K_{0}^{2}} \int_{0}^{z} 2 K_{0} \Delta K f\left(z^{\prime}\right) d z^{\prime},
$$

where $k_{u}=2 \pi / \lambda_{u}, f(z)$ describes the error shape, $0<$ $|f(z)| \leq 1 . f(z)$ is a periodic function with period length $\lambda_{\delta}$ and $f(z)=f\left(z+\lambda_{\delta}\right)$. If $z$ is normalized, $z_{n}=z / \lambda_{\delta}$, Eq. (3) becomes

$$
\begin{aligned}
\Delta \varphi\left(z_{n}\right) & =\frac{k_{u}}{1+K_{0}^{2}} 2 K_{0} \Delta K \lambda_{\delta} \int_{0}^{z_{n}} f\left(z_{n}^{\prime}\right) d z_{n}^{\prime} \\
& =\frac{2 k_{u} K_{0}^{2}}{1+K_{0}^{2}} \frac{\Delta K}{K_{0}} \lambda_{\delta} g\left(z_{n}\right) .
\end{aligned}
$$

Since the error function is periodic, the integration can be restricted to one error period length. A SASE-FEL always radiates at a wavelength for which $\langle\Delta \varphi\rangle=0$. Therefore the rms phase shake is given by

$$
\begin{aligned}
\sigma_{\Delta \varphi} & =\frac{2 k_{u} K_{0}^{2}}{1+K_{0}^{2}} \frac{\Delta K}{K_{0}} \lambda_{\delta} \sqrt{\int_{0}^{1} g^{2}\left(z_{n}\right) d z_{n}} \\
& =\alpha \frac{2 k_{u} K_{0}^{2}}{1+K_{0}^{2}} \frac{\Delta K}{K_{0}} \lambda_{\delta} .
\end{aligned}
$$

This equation is valid for all kinds of periodic errors. Different gap profiles are described by the coefficient $\alpha$. The strength is given by $\Delta K$. Table II lists $\alpha$ for different profiles.

The rms phase shake defined in Eq. (5) is proportional to the product of $\Delta K / K$ and $\lambda_{\delta}$. This is illustrated in Fig. 2. Figure 2(a) shows the $K$ variation along the undulator. The product of $\Delta K / K$ and $\lambda_{\delta}$ for the black and red curves are the same. The corresponding power gain curves are shown in Fig. 2(b). They almost coincide resulting in almost identical power reduction even though $\Delta K / K$ of the red curve is 5 times that of the black curve. In contrast the product for the blue curve, which has the same $\Delta K / K$ as the black curve, is 2 times larger. The comparison with the other curves in Fig. 2(b) shows the reduced growth rate.

A simple physical explanation is given below: For nonsteering errors and small error periods on the order of a gain length, the perturbation of ponderomotive phase is proportional to the perturbation of the $K$ parameter and the

TABLE II. Coefficient $\alpha$ for different error geometries.

\begin{tabular}{lccccc}
\hline \hline Error Type & Sinus & Sawtooth & Triangle & Constant & Parabolic \\
\hline Coefficient $\alpha$ & $\sqrt{2} / 4 \pi$ & $1 / 6 \sqrt{5}$ & $1 / 2 \sqrt{30}$ & $1 / 4 \sqrt{3}$ & $3 \sqrt{6 / 35}$ \\
\hline \hline
\end{tabular}



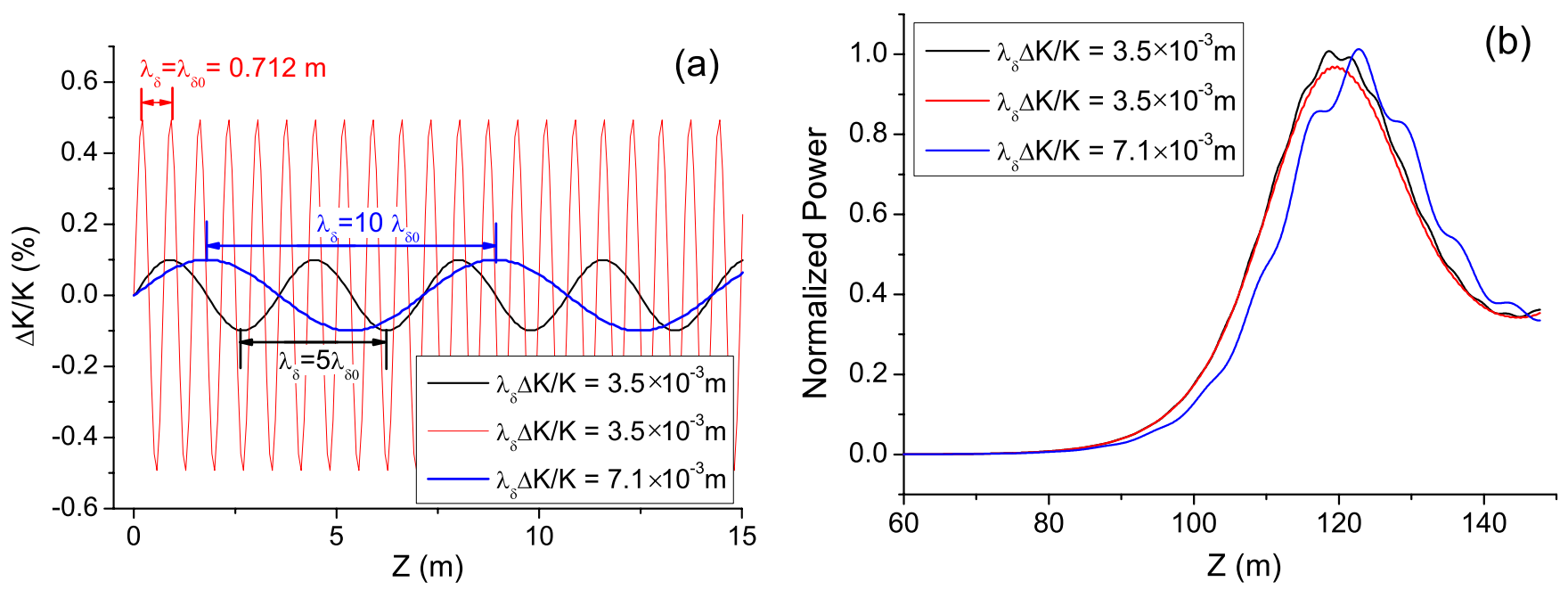

FIG. 2. (Color) Impact of three different sinus-type errors on power degradation. Different error amplitudes and period lengths are shown in (a). The product $\Delta K / K$ and $\lambda_{\delta}$ of the red and black curves are the same, that of the blue one is 2 times larger. The normalized power curves simulated with GENESIS 1.3 shown in (b) with the same phase shake (red and black curves) have very similar power growth. In contrast, the blue curve shows a slower growth rate leading to a longer saturation length.

length over which it is applied, thus leading to the form of Eq. (5). On the other hand, the criterion $\Delta K / K<\rho$ can be considered as an asymptote. In this case, saturation is reached before a significant deviation of ponderomotive phase can be obtained, and thus there is no power degradation. This is in qualitative agreement with Ref. [12].

\section{Compensation of linear temperature variation}

Over the length of an undulator system even a small temperature gradient may accumulate to several degrees. Because of the reversible temperature coefficient of the

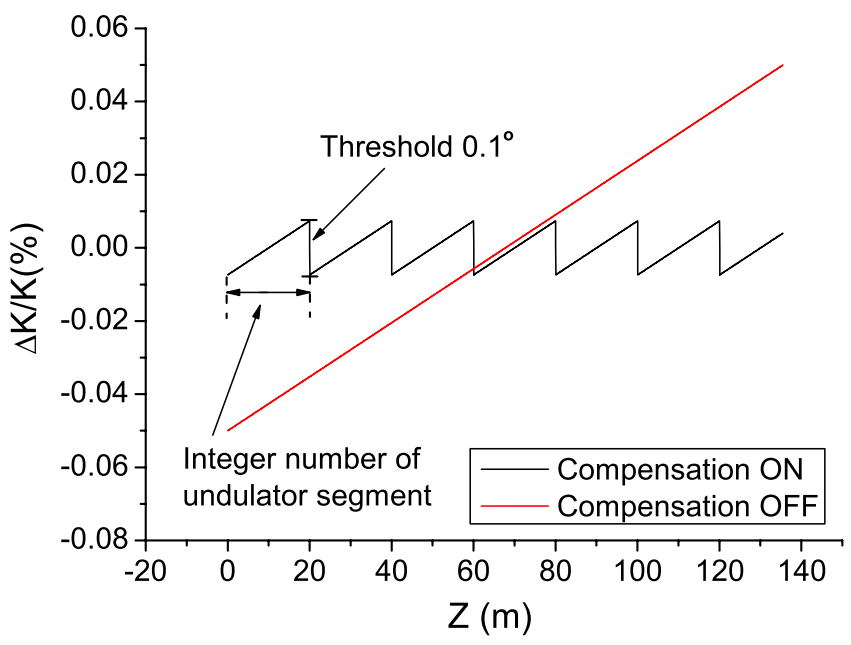

FIG. 3. (Color) Temperature compensation by gap adjustment. Red: The $\Delta K / K$ variation along the undulator for the uncompensated case. A value of $0.1 \%$ corresponds to $-1^{\circ} \mathrm{C}$. Black: After gap compensation in seven steps the remaining uncompensated part is a periodic sawtooth function that can be treated using Eq. (5) and Fig. 6.
$\mathrm{NdFeB}$ magnet material used in the undulator $(\eta=$ $-10^{-3} /{ }^{\circ} \mathrm{C}$ ), a $1{ }^{\circ} \mathrm{C}$ temperature increase corresponds to a reduction of the magnetic field in the undulator of $0.1 \%$ or vice versa. Such an error accumulated over the length of the undulator system would result in a large rms phase shake and corresponding power reduction. The gap of each undulator segment can be controlled separately. If the local temperature of the undulator is precisely known, a small gap correction can be applied to correct this field change.

The gap is corrected if a temperature variation larger than a threshold value is detected. This threshold value should correspond to the accuracy of the temperature measurement with typical values from 0.05 to $0.2^{\circ} \mathrm{C}$. Thus, after making this compensation, the resulting field variation corresponds to a periodic sawtooth error. The red curve in Fig. 3 shows the $\Delta K / K$ error resulting from a $1{ }^{\circ} \mathrm{C}$ linear temperature decrease along the undulator system. If, as shown, the gap is corrected in seven steps, a periodic sawtooth profile results. For demonstration, using Eq. (5) and the results of Table II, the rms phase shake can be calculated. A value of about $3^{\circ}$ is obtained. For phase shake this is already a small value having very little effect on FEL performance, as will be verified in the next section. However, it could be made even smaller by using more steps. If each undulator segment is adjusted, 33 steps (sawteeth) are possible if there is accurate temperature information available.

\section{NUMERICAL SIMULATION}

Extended simulations using GENESIS 1.3 [18] have been made. One reason for these simulations was to numerically study the influence of periodic errors on power degradation. A second reason was to confirm and reevaluate the 
error budget for the XFEL. FEL power was calculated at a fixed length, which is still in the exponential gain regime but already close to the saturation point. For further analysis, the power of an error configuration is normalized to the power obtained for an ideal undulator.

\section{A. Power reduction due to phase shake}

Unidirectional and alternating phase shift errors were studied in a similar fashion as mentioned in the last section. The results for SASE1 are shown in Fig. 4. At a given value for the phase shifter error, alternating errors show stronger power degradation than unidirectional error. This is consistent with the analysis in Sec. II A. It is also seen in Fig. 4 that, as long as the phase error is less than $10^{\circ}$, the power degradation is below $10 \%$ for both types. This is considered acceptable. What cannot be explained by the analysis in Sec. II A is the difference between a positive and negative phase error in case of the unidirectional phase shift. The different power reduction between the two is caused by a small difference in the wavelength (see Ref. [16]). A positive phase shift gives rise to lasing at a slightly longer wavelength which in turn leads to a larger power extraction from the electron beam close to saturation.

As described by Eq. (5), the rms phase shake is proportional to the product of undulator error amplitude $\Delta K$ and period $\lambda_{\delta}$. Since the power reduction corresponds to the rms phase shake, the same power reduction can be expected for different combinations of $\Delta K$ and $\lambda_{\delta}$, as long as their product is constant. For verification, numerical simulations with GENESIS 1.3 have been performed. The results obtained are shown in Fig. 5, which illustrates curves of constant power reduction from $10 \%$ to $40 \%$, using sinustype errors. The curves are best fits to the function $\Delta K / K=a \lambda_{\delta}^{b}$. It can be seen that for all curves $b \approx-1$, thus verifying the assumption. Moreover, there are four

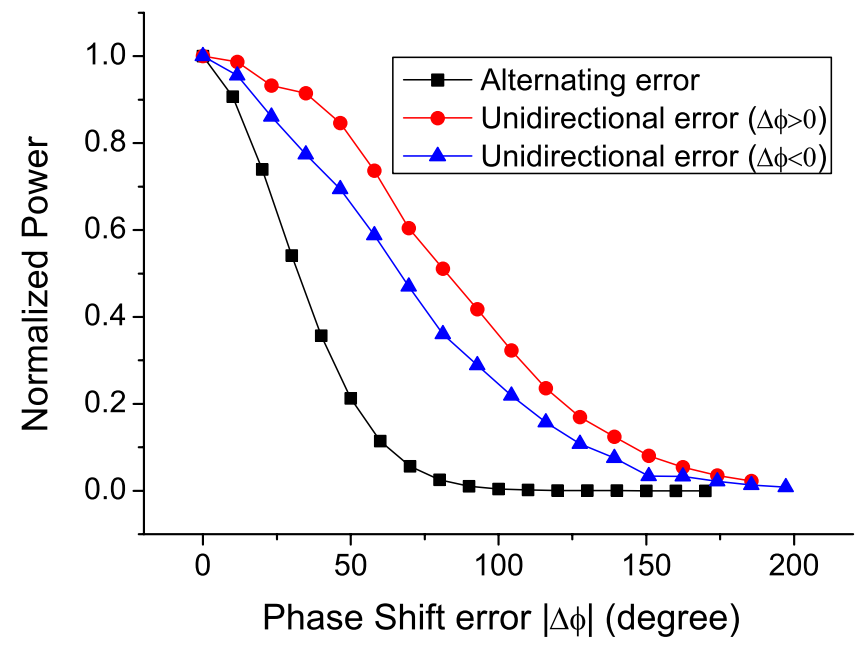

FIG. 4. (Color) Comparison of power degradation by unidirectional and alternating phase shift error. The normalized power is plotted against phase errors.



FIG. 5. (Color) Curves of constant power reduction for different error periods $\lambda_{\delta}$ and amplitudes $\Delta K$. The results to a fit $\Delta K / K=a \lambda_{\delta}^{b}$ are also shown. $b \approx-1$ verifies that constant power reduction corresponds to the product of $\Delta K / K$ and $\lambda_{\delta}$.

support points with a distance of $1.2 \mathrm{~m}$ on a 5-m-long SASE1 undulator segment. Figure 5 shows that for this error period, the error in $\Delta K / K$ can be as large as $0.366 \%$ for a power reduction of $\leq 10 \%$. This relaxes the requirement for girder deformation significantly as compared to previous work [1].

The correlation between rms phase shake and power reduction is shown in Fig. 6(a). Periodic sinus, sawtooth, triangle, and stepwise constant errors are shown. The phase shifter errors shown in Fig. 4 are included as well. Within only small scatter they all lie on one universal curve as can be seen in Fig. 6. It is evident that there is a very strong correlation between rms phase shake and power degradation. Unidirectional phase shift errors play a special role because they shift the resonance wavelength. Therefore their power degradation is less.

In reality there are random taper errors, which, for example, might be induced by limited motion control accuracy. These are clearly long-range random errors, which affect an undulator segment. In order to study their influence a sawtooth error was chosen. Its amplitude for $\Delta K / K$ was varied in a random fashion from $0.15 \%$ up to $0.45 \%$.

In Fig. 6(b), 900 simulations for different taper configurations are shown. The periodic sawtooth results are included for comparison. In both cases the power decreases as the rms phase shake increases. The random error result shows a much larger scatter. On average, the power degradation seems to be approximately the same.

\section{B. Periodic and random errors}

In reality, different error types are combined. For a given gap, the girder deformation for all undulator segments is identical and is strictly periodic. The gap taper, however, 



FIG. 6. (Color) Correlation between power reduction and rms phase shake for periodic (a) and random (b) errors. In (a), the universal behavior for periodic undulator errors as discussed in the text is shown. Alternating and unidirectional phase shifter errors are included for comparison. In (b), the comparison of the correlation of rms phase shake and power reduction for periodic (red) and random (black) sawtooth errors is shown. Nine hundred random configurations were calculated. Note the different scales in (a) and (b).

contributes a random component. Therefore, both errors were combined in the following simulation. In order to make a conservative assumption, the maximum periodic girder deformation is set to $10 \mu \mathrm{m}$ and the maximum taper error is set to $1 \mu \mathrm{m}$. The gap taper was varied randomly over the undulator system. Figure 7(a) shows the normalized power as a function of the rms phase shake. As random errors are included, there is quite some scatter in the results. One sees that there are configurations where the maximum observed power reduction is up to $\approx 18 \%$ but there are also some with power gain up to $8 \%$. In a similar fashion, the rms phase shake extends from 0.06 to $0.18 \mathrm{rad}$ over a range of $0.12 \mathrm{rad}$ or about 7 degrees. Because of their random character, these results need now to be considered statistically. Figure 7(b) shows the density plot of the normalized power averaged over all phase shakes. The center is shifted down by $3 \%$. The statistical analysis shows that, for the parameters given, more than $95 \%$ of the investigated cases suffer from a power loss such that the normalized power is above 0.88 . The statistical probability for larger losses is small, only $2.5 \%$. This is considered a sufficiently safe design.

Note that in Figs. 6 and 7 the normalized power sometimes exceeds unity, indicating that it is higher than in the
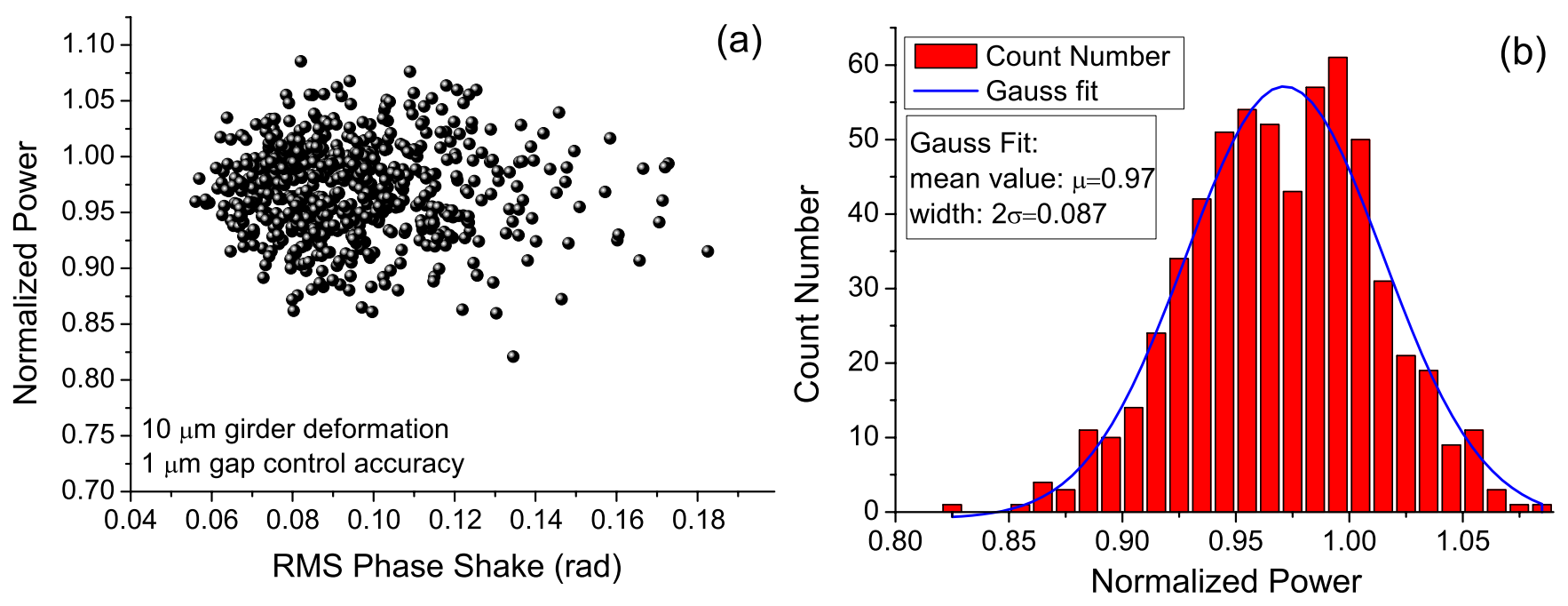

FIG. 7. (Color) The power degradation due to combined periodic sinus and random sawtooth error calculated for 600 different configurations shown versus phase shake (a) and as probability distribution (b). The density plot (b) has been fitted assuming a Gaussian distribution. The center is shifted down by $3 \%$. The $95 \%$ confidence level of \pm 2 standard deviations starts at 0.88 . The statistical probability for higher losses is only $2.5 \%$ and considered acceptable. 


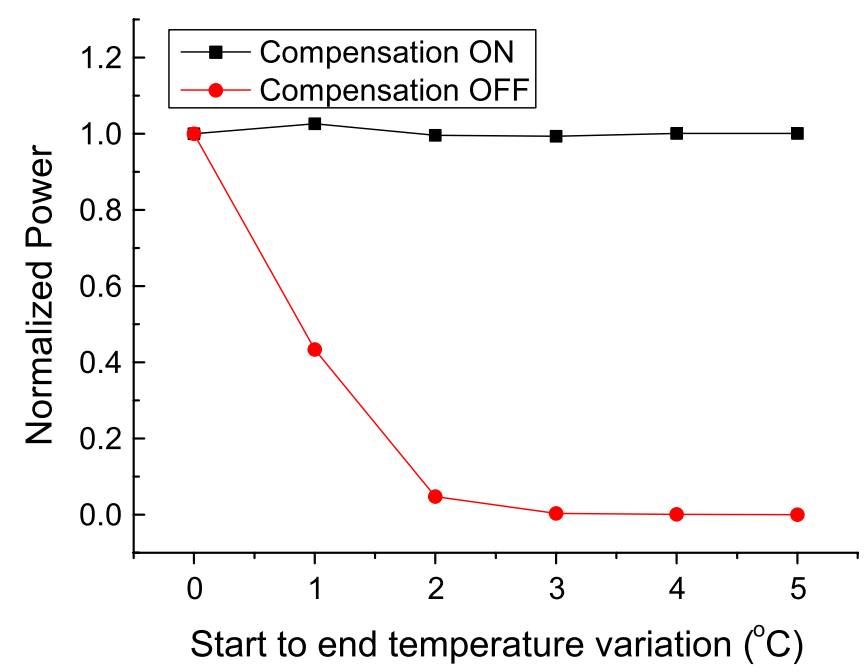

FIG. 8. (Color) The black curve shows the normalized power for the uncompensated case for start-to-end variations up to $0.5 \%$ corresponding to $5^{\circ} \mathrm{C}$. The red curve shows the normalized power when the gap compensation is used. All power losses can be recovered.

case without errors. The reason is that, for some random distributions, the $K$ value at the point where the results were evaluated is smaller and the error acts as a taper. In a number of cases, this has been verified by checking and comparing the details of these simulations.

\section{Compensation of a temperature gradient}

As already mentioned in Sec. IIC and illustrated in Fig. 3, a temperature gradient, i.e., a linear increase or decrease of temperature along the undulator system can be compensated by a local gap correction. Figure 8 shows the impact on power reduction as calculated using GENESIS 1.3. The normalized power is shown as a function of the total $\Delta K / K$ change over the undulator system. For the uncompensated case (black curve), a start-to-end $\Delta K / K$ of $0.1 \%$ corresponds to $1{ }^{\circ} \mathrm{C}$ and leads to a power reduction by more than $50 \%$ (first data point). For $\Delta K / K$ larger than $0.2 \%$ there is almost no radiated power. If the gap compensation is turned on (red curve), practically all losses can be recovered. Using this temperature compensation scheme, even a $5^{\circ} \mathrm{C}$ temperature variation can be compensated. However, it should be noted that a precise local temperature measurement at each undulator segment is needed for the correction in order to have accurate gap compensation.

\section{CONCLUSION}

A thorough study of tolerances for the SASE1 undulator system for the European XFEL was made. Different types of errors encountered in a realistic undulator system were investigated. These included girder deformation changes due to changing magnetic load, gap taper due to limited accuracy of the motion control system, phase shifter errors, and the effect of temperature variation on the magnetic material. The ponderomotive phase shake was calculated and compared with FEL power degradation results using GENESIS 1.3.

For XFEL parameters, we verified numerically that there is a close correlation between the rms phase shake and FEL power degradation, which is independent of a specific error distribution. If these errors are periodic, the correlation is very close, while for randomly distributed errors there is more scatter in the results, but on average there is agreement as well. For the parameters used for the European XFEL, the power degradation induced by a specific periodic error model can thus be estimated quickly without using FEL codes. This method was applied to reevaluate hardware-relevant aspects of the error budget for the SASE1 undulator system for the European XFEL. The results show that some cost-driving specifications can be relaxed to some extent as compared to earlier studies. In order to stay within a power loss not more than $\approx 15 \%$ girder deformation should be limited to $\leq 10 \mu \mathrm{m}$. Girder position should be controlled to $\leq 1 \mu \mathrm{m}$, and the phase adjustment to better than $10^{\circ}$. Applying the methods of this paper, an elegant way was found to replace the stringent temperature requirements over the whole undulator system by a requirement to limit the temperature gradient over one undulator segment and use of local gap corrections. These results will have a large impact on the design of the XFEL undulator systems.

[1] M. Altarelli et al., The European X-Ray Free-Electron Laser Technical Design Report, ISBN 3-935702-17-5.

[2] A. M. Kondratenko and E. L. Saldin, Part. Accel. 10, 207 (1980).

[3] R. Bonifacio, C. Pellegrini, and L. M. Narducci, Opt. Commun. 50, 373 (1984).

[4] J. Pflueger, in Proceedings of the 27th International Free Electron Laser Conference, 2005, TUOC002, p. 378.

[5] B. L. Bobbs et al., Nucl. Instrum. Methods Phys. Res., Sect. A 296, 574 (1990).

[6] B. Faatz, J. Pflueger, and Y. M. Nikitina, Nucl. Instrum. Methods Phys. Res., Sect. A 393, 380 (1997).

[7] E. Gluskin, N. A. Vinokurov, G. Decker, R. J. Dejus, P. Emma, P. Illinski, E. R. Moog, H. D. Nuhn, and I. B. Vasserman, Nucl. Instrum. Methods Phys. Res., Sect. A 475, 323 (2001).

[8] CDR of the LCLS Project, SLAC-R-593, 2002.

[9] Z. Huang and G. Stupakov, Phys. Rev. ST Accel. Beams 8, 040702 (2005).

[10] J. Pflueger and M. Tischer, Nucl. Instrum. Methods Phys. Res., Sect. A 483, 388 (2002).

[11] Y. Li, B. Faatz, and J. Pflueger, in Proceedings of the 29th International Free Electron Laser Conference, 2007, WEPPH005, p. 326.

[12] L. H. Yu, S. Krinsky, R. L. Gluckstern, and J. B. J. van Zeijts, Phys. Rev. A 45, 1163 (1992). 
[13] R. P. Walker, Sincrotrone Trieste ST/M-93/3, 1993.

[14] H. P. Freund and R. H. Jackson, Nucl. Instrum. Methods Phys. Res., Sect. A 331, 461 (1993).

[15] A. Friedman, S. Krinski, and L. H. Yu, IEEE J. Quantum Electron. 30, 1295 (1994).

[16] Y. Li, B. Faatz, and J. Pflueger, TESLA-FEL Report
No. 2007-07, DESY, Hamburg.

[17] J. Pflueger, H. Lu, and T. Teichmann, Nucl. Instrum. Methods Phys. Res., Sect. A 429, 386 (1999).

[18] S. Reiche, Nucl. Instrum. Methods Phys. Res., Sect. A 429, 243 (1999). 